\title{
A Variational Framework for Single Image Dehazing
}

\author{
Adrian Galdran ${ }^{1}$, Javier Vazquez-Corral ${ }^{2}$, David Pardo ${ }^{3}$, Marcelo Bertalmío ${ }^{2}$ \\ ${ }^{1}$ Tecnalia Research \& Innovation, Basque Country, Spain \\ ${ }^{2}$ Departament de Tecnologies de la Informació i les Comunicacions, Universitat \\ Pompeu Fabra, Barcelona, Spain \\ ${ }^{3}$ University of the Basque Country (UPV/EHU) and IKERBASQUE (Basque \\ Foundation for Sciences), Bilbao, Spain
}

\begin{abstract}
Images captured under adverse weather conditions, such as haze or fog, typically exhibit low contrast and faded colors, which may severely limit the visibility within the scene. Unveiling the image structure under the haze layer and recovering vivid colors out of a single image remains a challenging task, since the degradation is depth-dependent and conventional methods are unable to handle this problem.

We propose to extend a well-known perception-inspired variational framework [1] for the task of single image dehazing. The main modification consists on the replacement of the value used by this framework for the grey-world hypothesis by an estimation of the mean of the clean image. This allows us to devise a variational method that requires no estimate of the depth structure of the scene, performing a spatially-variant contrast enhancement that effectively removes haze from far away regions. Experimental results show that our method competes well with other stateof-the-art methods in typical benchmark images, while outperforming current image dehazing methods in more challenging scenarios.
\end{abstract}

Keywords: Image dehazing, Image defogging, Color correction, Contrast enhancement

\section{Introduction}

The effect of haze in the visibility of far away objects is a well-known physical property that we perceive in different ways. For example, an object loses contrast as its depth in the image increases, and far away mountains present a bluish tone [8]. Haze is produced by the presence of suspended little particles in the atmosphere, called aerosols, which are able to absorb and scatter the light beams. Aerosols can range from small water droplets to dust or pollution, depending on their size. Scientific models of the propagation of light under such conditions began with the observation of Koschmieder [14]. He stated that a distant object tends to vanish by the effect of the atmosphere color, which replaces the color of the object. Consequently, Koschmieder established a simple linear relationship between the luminance reflected by the object and the luminance reaching the 
observer. This linear relationship is based on the distance between the observer and the object. From then on, the study of interaction of light with the atmosphere as it travels from the source to the observer has continued growing as a research area in applied optics $[17,16]$.

Restoring images captured under adverse weather conditions is of clear interest in both image processing and computer vision applications. Many vision systems operating in real-world outdoor scenarios assume that the input is the unaltered scene radiance. These techniques designed for clear weather images may suffer under bad weather conditions where, even for the human eye, discerning image content can represent a serious challenge. Therefore, robustly recovering visual information in bad weather conditions is essential for several machine vision tasks, such as autonomous robot/vehicle navigation [10] or video surveillance systems $[32,29]$. Aerial and remotely sensed images, related to applications as land cover classification $[34,15]$, can also benefit from efficient dehazing techniques.

As Koschmieder stated, the problem of restoring true intensities and colors (sometimes referred to as albedo) presents an underlying ambiguity that cannot be analytically solved unless scene depth data is available [23]. For this reason, most of the previous approaches rely on physically-based analytical models of the image formation. The main goal of these approaches is to estimate the transmission, (or alternatively the depth) of the image to estimate the transmission of the image, that describes the part of the light that is not scattered and reaches the camera, and later on, to obtain the albedo based on the transmission. Alternatively, depth can also be estimated. These approaches can be later divided into multiple images ones $[22,18,20,19,21]$, or single image ones $[12,6]$. On the other hand, there are also works that compute the albedo in the first place and obtain a depth map as a by-product. In [30], Tan estimates the albedo by imposing a local maximization of contrast, while in [5] Fattal assumes that depth and surface shading are uncorrelated. Unfortunately, both methods rely on the assumption that depth is locally constant, and the obtained images suffer from artifacts and are prone to over-enhancing.

Regarding all the previously stated, contrast enhancement of hazy images seems to be a straight-forward solution for this problem. However, conventional contrast enhancement techniques such as histogram equalization are not applicable due to the spatially variant nature of the degradation. Fortunately, recent research has presented more advanced contrast enhancement techniques that can successfully cope with spatially inhomogeneous degradations such as the one produced by haze. In this work, we rely on the perceptually inspired color enhancement framework introduced by Bertalmio et al. [1]. We propose to replace the original grey-world hypothesis by a rough estimate of the mean value of the haze-free scene. This value is softly based on Koschmieder statement [14]. A different modification of this hypothesis was already performed in previous works $[7,33]$.

The rest of the paper is structured as follows. In the following section we review recent methods for image dehazing. Next, we formulate the image de- 
hazing problem in a variational setting. Section 4 is devoted to experimental results and comparison to other state-of-the-art methodologies. We end up in section 5 by summarizing our approach and discussing possible extensions and improvements.

\section{Background and Related Work}

Most of the previous work on image dehazing is based on solving the image formation model presented by Koschmieder [14] that can be computed channelwise as follows

$$
\mathrm{I}(x)=\mathrm{t}(x) \mathrm{J}(x)+(1-\mathrm{t}(x)) \mathrm{A},
$$

where $x$ is a pixel location, $\mathrm{I}(x)$ is the observed intensity, $\mathrm{J}(x)$ is the scene radiance, corresponding to the non-degraded image, transmission $\mathrm{t}(x)$ is a scalar quantity that is inversely related to the scene's depth and is normalized between 0 and 1 ,, while A, known as airlight, plays the role of the color of the haze, which is usually considered constant over the scene, and therefore in a channel-wise formulation it is a scalar value. Solving Eq. (1) is an under-constrained problem, i.e. there are a large number of possible solutions. To constrain this indeterminacy, extra information in different forms has been introduced in the past. For example, in [19] several instances of the same scene acquired under different weather conditions are employed to obtain a clear image. The near infra-red channel is fused with the original image in [27], and the work in [13] retrieves depth information from geo-referenced digital models, while in [28] multiple images taken through a polarizer at different orientations are used. Unfortunately, this extra information is often unavailable, difficulting the practical use of these techniques.

Dehazing is particularly challenging when only a single input image is available. In this case, the majority of existing methods are also focused on solving Eq. (1) by inferring depth information based on different means. In [4], assumptions on the geometry of hazy scenarios are made. Tarel et al. [31] estimate the atmospheric veil (equivalent to the depth map) through an optimization procedure in which they impose piecewise smoothness. The dark channel methodology [12], probably the most successful technique to date, is based on the statistical observation that haze-free images are colorful and contain textures and shadows, therefore lacking locally the presence of one of the three color components. On the contrary, hazy images present less contrast and saturation. As depth increases and the haze takes over the image, the contrast and saturation further decrease providing an estimate of the depth information based on which it becomes possible to invert Eq. (1), obtaining high-quality results. More recently, Fattal [6] elaborates on a local model of color lines to dehaze images.

Several methods that are independent of an initial estimation of the scene depth have also been devised. Tan [30] imposes a local increase of contrast in the image and a similar transmission value for neighboring pixels. Fattal [5] separates the radiance from the haze by assuming that surface shading and scene transmission are independent. Nishino et al. [23] do not compute depth 
in an initial stage, but rather estimate it jointly with the albedo in a Bayesian probabilistic framework.

\section{$3 \quad$ Variational Image Dehazing}

The majority of current dehazing algorithms are based on an estimation of the image depth (or transmission). Therefore, these methods are susceptible to fail when the physical assumptions underlying Eq. (1) are violated. This is a common phenomena both in real life, for example, when there is a source of light hidden by the haze, and in virtually-generated images that add different types of fog. Methods that do not estimate the model depth do not suffer from this problem, but they usually result in over-enhanced images due to the special characteristics of the degradation associated with haze. More conventional contrast enhancement algorithms, such as histogram equalization, are not suitable either. Fortunately, recent spatially-variant contrast enhancement techniques can be adapted to perform well for image dehazing tasks. In the following, we develop a variational framework for image dehazing that enforces contrast enhancement on hazy regions of the image throughout an iterative procedure allowing us to control the degree of restoration of the visibility in the scene.

\subsection{Variational Contrast Enhancement}

In 2007, Bertalmío et al. [1] presented a perceptually-inspired variational framework for contrast enhancement. Their method is based on the minimization of the following functional for each image channel $I$ :

$$
E(\mathrm{I})=\frac{\alpha}{2} \sum_{x}\left(\mathrm{I}(x)-\frac{1}{2}\right)+\frac{\beta}{2} \sum_{x}\left(\mathrm{I}(x)-\mathrm{I}_{0}(x)\right)^{2}-\frac{\gamma}{2} \sum_{x, y} \omega(x, y)|\mathrm{I}(x)-\mathrm{I}(y)|,
$$

where $I$ is a color channel (red, green or blue) with values in $[0,1], I_{0}$ is the original image, $x, y$ are pixel coordinates, $\alpha, \beta, \gamma$ are positive parameters and $\omega(x, y)$ is a positive distance function with value decreasing as the distance between $x$ and $y$ increases. This method extends the idea of variational contrast enhancement presented by Sapiro and Caselles [26] and it also shows a close connection to the ACE method [25]. Bertalmío and co-authors have later revealed connections between this functional and the human visual system: they generalized it to better cope with perception results [24], and they established a very strong link with the Retinex theory of color $[3,2]$.

The minimization of the image energy in Eq. (2) presents a competition between two positive terms and a negative one. The first positive term aims at preserving the gray-world hypothesis, by penalizing deviation of $\mathrm{I}(x)$ from the $1 / 2$ value, while the second positive term prevent the solution from departing too much from the original image, by restricting output values to be relatively close to the initial $\mathrm{I}_{0}(x)$. The negative competing term attempts to maximize 
the contrast. Focusing on this negative term of Eq. (2) we can observe a very useful relation with dehazing methods. It can be written as:

$$
\sum_{x, y} \omega(x, y)|\mathrm{I}(x)-\mathrm{I}(y)|=\sum_{x, y} \omega(x, y)(\max (\mathrm{I}(x), \mathrm{I}(y))-\min (\mathrm{I}(x), \mathrm{I}(y))) .
$$

We can now see that the contrast term is maximized whenever the minimum decreases or the maximum increases, corresponding to a contrast stretching. Notice that the first case, i.e., the minimization of local intensity values, is one of the premises of a haze-free image, according to the Dark Channel prior [11].

\subsection{Modified Gray-World Assumption}

In the image dehazing context, the Gray World hypothesis implemented in Eq. (2) is not adequate, since we want to respect the colors of the haze-free image, not to correct the illuminant of the scene. Therefore, to approximately predict which should be the mean value of a dehazed scene, we rely on the model of Eq. (1), that we rewrite here in terms of the luminance of each channel:

$$
\mathrm{L}^{j}=\mathrm{L}_{0}^{j} \mathrm{t}+(1-\mathrm{t}) A^{j},
$$

where $j \in\{R, G, B\}$. By rearranging, taking the average of each term and assuming that $\mathrm{L}$ and $\mathrm{t}$ are independent, we arrive to:

$$
\operatorname{mean}\left(\mathrm{L}_{0}^{j}\right) \cdot \operatorname{mean}(\mathrm{t})=\operatorname{mean}\left(\mathrm{L}^{j}\right)+(\operatorname{mean}(\mathrm{t})-1) \operatorname{mean}\left(\mathrm{A}^{j}\right) .
$$

Now, let us assume that the image presents regions at different depth distances, therefore, the histogram of depth values will be approximately uniformly distributed. In this way, we can set mean $(\mathrm{t})=\frac{1}{2}$ and approximate the previous equation by:

$$
\frac{\operatorname{mean}\left(\mathrm{L}_{0}^{j}\right)}{2} \approx \operatorname{mean}\left(\mathrm{L}^{j}\right)+\left(\frac{1}{2}-1\right) \operatorname{mean}\left(\mathrm{A}^{j}\right) .
$$

Let us note again that the airlight $\mathrm{A}$ is a constant value for each channel, that can be roughly approximated by the maximum intensity value on each channel, since haze regions are usually those with higher intensity.. Thus, a reasonable approximation for the mean value of the haze-free scene is given by:

$$
\mu^{j}=\operatorname{mean}\left(\mathrm{L}_{0}^{j}\right) \approx 2 \operatorname{mean}\left(\mathrm{L}^{j}\right)-\mathrm{A}^{j} .
$$

We then rewrite the energy functional as:

$$
E\left(\mathrm{I}^{j}\right)=\frac{\alpha}{2} \sum_{x}\left(\mathrm{I}^{j}(x)-\mu^{j}\right)+\frac{\beta}{2} \sum_{x}\left(\mathrm{I}^{j}(x)-\mathrm{I}_{0}^{j}(x)\right)^{2}-\frac{\gamma}{2} \sum_{x, y} \omega(x, y)\left|\mathrm{I}^{j}(x)-\mathrm{I}^{j}(y)\right| .
$$

To minimize the above energy we first need to compute its Euler-Lagrange derivative. Close details about the computation of the variational derivatives of 
the different terms are given in [1], where the authors find that a minimizer of (8) must satisfy:

$$
\delta E\left(\mathrm{I}^{j}\right)=\alpha\left(\mathrm{I}^{j}(x)-\mu_{j}\right)+\beta\left(\mathrm{I}^{j}(x)-\mathrm{I}_{0}^{j}(x)\right)-\gamma R\left(\mathrm{I}^{j}\right)(x)=0, \quad j \in\{R, G, B\}
$$

where the function $R(\mathrm{I})$ is a contrast enhancement operator:

$$
R(I)(x)=\frac{\sum_{y} \omega(x, y) s(\mathrm{I}(x)-I(y))}{\sum_{y} \omega(x, y)},
$$

and $s$ is a smooth approximation of the sign function, that accounts for the first derivative of the absolute value.

We can now apply a gradient descent strategy. To this end, we solve $\frac{\delta I}{\delta t}=$ $-\delta E(I)$, being $t$ the evolution parameter. For the case of the energy given by Eq. (8), with the modified gray world assumption, after an explicit discretization in time, we have:

$$
\mathrm{I}_{k+1}^{j}=\mathrm{I}_{k}^{j}(1-\Delta t(\beta+\gamma))+\Delta t\left(\beta \mu^{j}+\gamma \mathrm{I}_{0}^{j}\right)+\Delta t\left(\eta R\left(\mathrm{I}_{k}^{j}\right)\right), \quad j \in\{R, G, B\}
$$

The initial condition for this descent is the input image $\mathrm{I}_{k=0}=\mathrm{I}(x)$. The computations of the operator $R$ are reformulated in terms of convolutions and computed using Fast Fourier Transforms. This brings a computational improvement to the method, since the effort to compute expression (10) falls down from $\mathcal{O}\left(N^{2}\right)$ to $\mathcal{O}(N \log (N))$. Details of this argument for complexity reduction can be found in [1].

\section{Experimental Results}

In this section we compare our proposed method versus current state-of-theart results. In recent years, very powerful algorithms for image dehazing have appeared. When processing typical benchmark images, it is very hard to retrieve more visibility or natural colors than these methods already do although our method shows a competitive behavior in these images. However, when dealing with more realistic images, in which the fog does not follow a linear model such as Eq. (1) or the illumination is uneven, we have observed that the majority of the state-of-the-art methods tends to create severe artifacts, while ours is still able to dehaze these scenes without introducing this kind of corruption.

Let us also notice that, due to the difficulty to obtain ground-truth information of hazy/haze-free images, evaluation of dehazing methods has usually a subjective component. Quality of the results should be measured by the plausibility of the restored colors, as well as the recovered visibility in far away areas of the scene. To evaluate our results, we have run Eq. 11 with the following values: $\alpha=\beta=0.5, \gamma$ varied depending on the amount of enhancement we desired, $\eta$ was set to 0 unless otherwise stated, and the distance function implemented was a Gaussian kernel with a standard deviation of 50 pixels. The time step was always $\Delta t=0.15$, and we considered that a steady-state of the gradient descent 


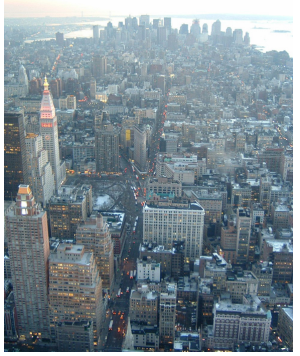

(a)

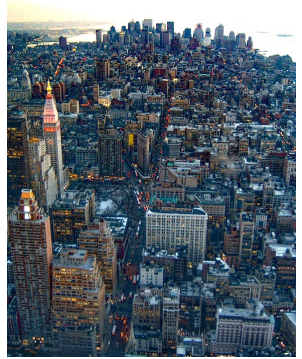

(e)

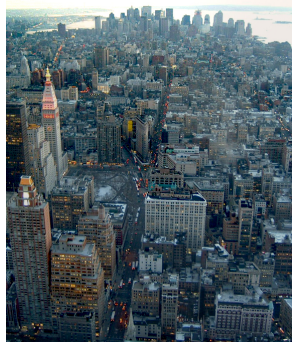

(b)

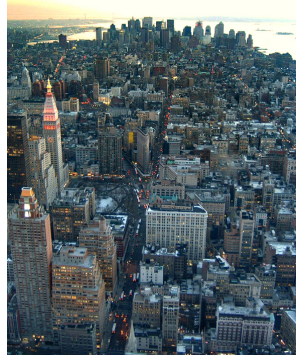

(f)

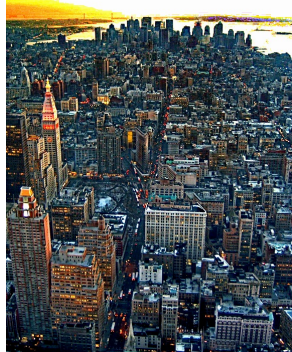

(c)

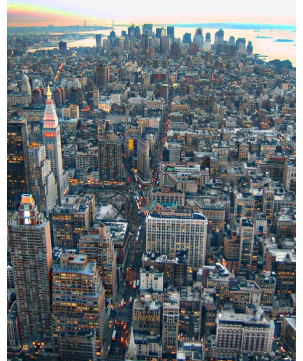

(g)

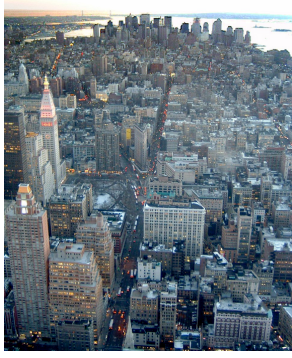

(d)



(h)

Fig. 1. (a) New York image (input). (b) to (h): Result of processing with methods of: (b) Fattal '08 [5] (c) Tan. '08 [30] (d) Kopf et al. [13] (e) Fattal '14 [6] (f) He et al. '11 [12] (g) Tarel et al. '09 [31] (h) Result of our method.

was achieved as soon as the Mean Square Error between one iteration and the next one falls below 0.02 . In all the experiments, we observed convergence within $6-12$ iterations. We have noticed that our method shows a strong robustness to slight parameter tuning.

Figure 1 displays an image of the city of New York, typically used for benchmarking of dehazing algorithms. Therein, our method is compared against the works in [5],[30], [13], [6], [12] and [31]. We see that visibility of distant objects is recovered by every method up to a reasonable degree. The very recent method of Fattal [6] and the powerful Dark Channel method [12] are possibly the ones recovering most vivid colors, although unfortunately they fail to recover the information underlying in the horizon (see upper right corner in Fig. (1e) and (1f)). Also, the method by Tan [30] suffers of noticeable over-saturation artifacts.

Figure 2 illustrates the performance of our method on an open scene image that contains a blue sky region. This is a challenging scenario for most dehazing algorithms. In fact, some of the methods perform an initial classification of pixels according to whether they belong to sky region or not. We see that only Kopf et al. method [13] maintains a natural appearance of the sky area. Unfortunately, this method needs geo-referenced data to work, and it is not usual to have 


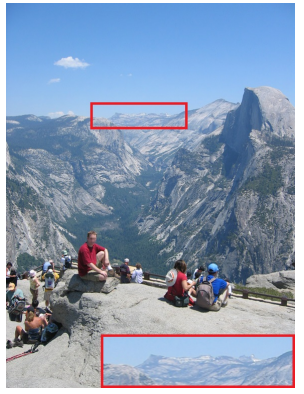

(a)

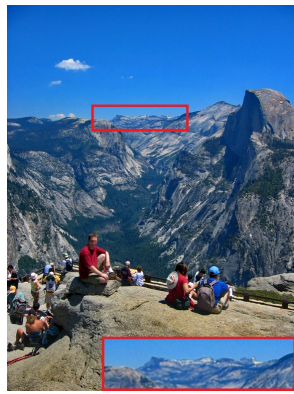

(e)

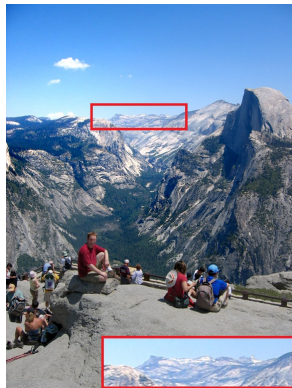

(b)

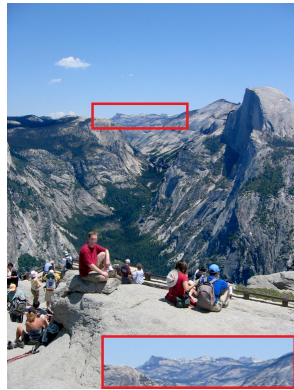

(f)

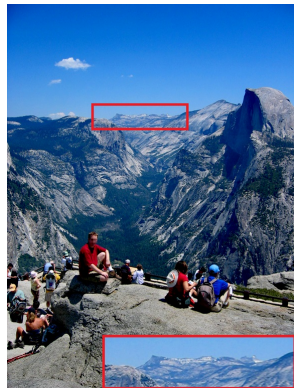

(c)

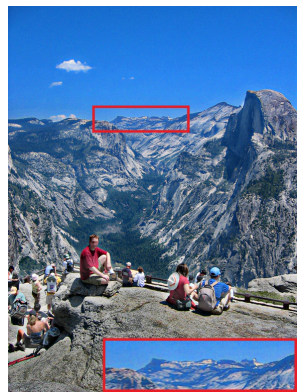

$(\mathrm{g})$

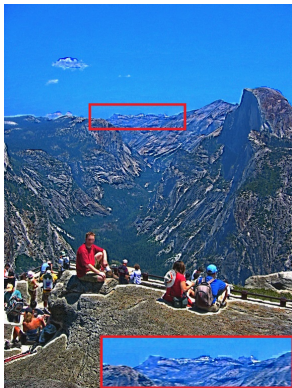

(d)



(h)

Fig. 2. a) Mountain image (input) b)-h) Result of processing with methods of b) Fattal '08 [5] c) Nishino et al. '12 [23] d) Tan '08 [30] e) He et al. '11 [12] f) Kopf et al. '08 [13] g) Tarel et al. '09 [31] h) Result of our method.

this kind of input at hand. The rest of the methods, including ours, tend to over-enhance sky regions. Regarding the elimination of the distant bluish haze surrounding mountain peaks, we provide magnified detail in the right bottom corner of each image in Fig. 2, where we can observe how only Kopf et al. method, together with Fattal [5] and our algorithm are able to suppress haze without introducing an unnatural blue in far-away regions.

The previous examples demonstrate how for typical images, existing algorithms (including ours) can handle haze in an adequate manner, recovering visibility up to a reasonable degree. On the other hand, each of the methodologies restore chromatic information in a different way, although the majority of the available techniques produce rather plausible colors. Differences in the performance are subtle and only little details reveal whether a method is performing better than another in particular regions of these particular images.

Unfortunately, little research has addressed the problem of image dehazing in a more challenging and realistic scenario, such as the one depicted in Fig. 3. Let us notice that most of the state-of-the-art methodologies rely on the previous computation of a depth map of the scene. They usually resort to a physical model of the image formation under haze and bad weather conditions, such as 


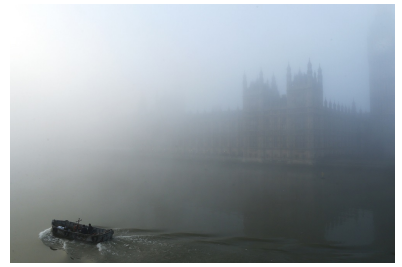

(a)

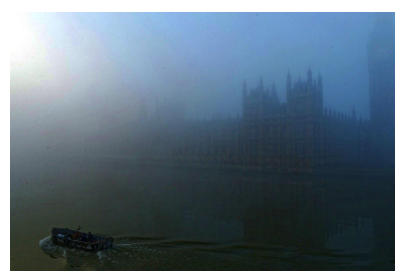

(d)

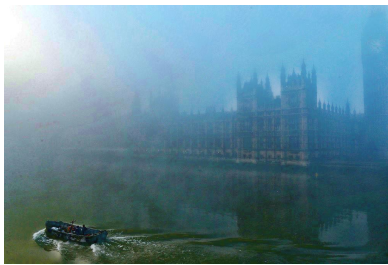

(b)



(e)

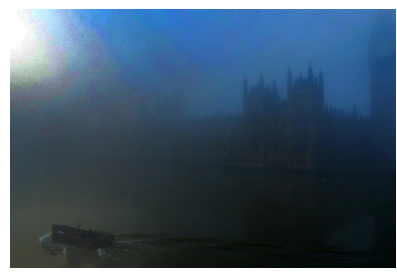

(c)

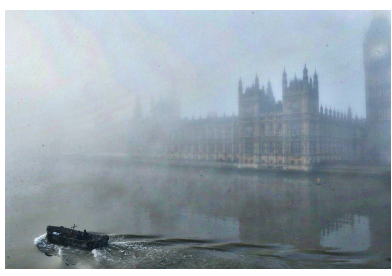

(f)

Fig. 3. a) Unevenly illuminated hazy image of the Thames river b)-f) Result of processing with methods of b) Tarel '09 [31] c) He et al. '11 [12] d) Gibson et al. '13 [9] e) Nishino et al. '12 [23] f) Result of our method.

Eq. (1). This model assumes illumination is constant in the scene. When this assumption is violated, the airlight cannot be considered to be constant. The result is a transmission underestimate or overestimate in unevenly illuminated areas, and color distortions characterized by dark blue regions appear in the restored images. This is the case for all the methods we tested, except for ours, that does not compute any depth information prior to restoration. Thanks to the attachment-to-data term, the strongly illuminated regions are handled properly, and scene structure can be recovered in the rightmost part of the image without introducing color distortion.

As a last example, let us notice that most works rely on the presence of enough chromatic information in the scene so as to recover the depth structure. When this chromatic information is weak or missing, the result is often an image with unpleasant color artifacts. Our method operates in a channel-wise manner, handling thus more robustly the lack of color cues in the input image, as can be appreciated in Fig. 4.

\section{Conclusions and Future Work}

In this paper, we have proposed a variational framework that can be used for image dehazing tasks. Extension of previous work on perceptual contrast enhancement allows us to devise a method that does not rely on an initial estimate of the depth information in the scene. Initial results of our method are promising, and comparable or better than state-of-the-art methods for typical benchmark 


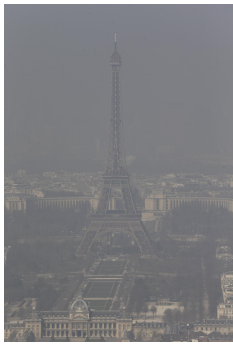

(a)

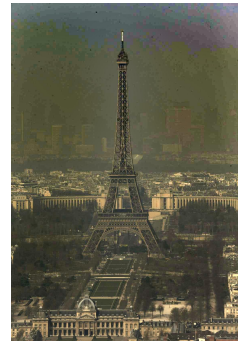

(b)

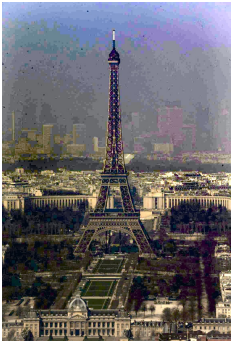

(c)

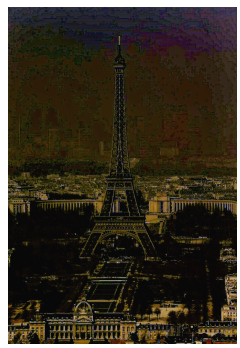

(d)

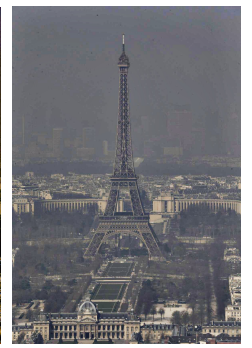

(e)

Fig. 4. a) An image of the city of Paris, with a lack of chromatic information due to haze in the scene b)-e) Result of processing with methods of b) He et al. '11 [12] c) Fattal '14 [6] d) Nishino et al. '12 [23] e) Result of our method.

images. Moreover, our algorithm performs well even in very adverse circumstances, such as unevenly illuminated scenes, where most methods are prone to introduce artifacts.

We are currently developing various extensions of our methodology, such as an image-fusion approach to image dehazing, in which the iterations of the gradient descent are fused to give an improved version of the dehazed image. Further work might consist on adding extra terms to the functional, for example, Eq. (2) could be combined with a depth map coming from any of the algorithms that are able to estimate $3 \mathrm{D}$ structure in the scene, to enforce denoising or deblurring tasks in far away areas of the scene.

\section{Acknowledgments}

JVC and MB were supported by European Research Council, Starting Grant ref. 306337, and by Spanish grants ref. TIN2011-15954-E and ref. TIN2012-38112.

\section{References}

1. Bertalmio, M., Caselles, V., Provenzi, E., Rizzi, A.: Perceptual color correction through variational techniques. IEEE Transactions on Image Processing 16(4), 1058-1072 (Apr 2007)

2. Bertalmio, M., Caselles, V., Provenzi, E.: Issues about retinex theory and contrast enhancement. Int J Comput Vis 83(1), 101-119 (Jun 2009)

3. Brian V. Funt, F.C.: Retinex in MATLAB. J. Electronic Imaging 13, 48-57 (2004)

4. Carr, P., Hartley, R.: Improved single image dehazing using geometry. In: Digital Image Computing: Techniques and Applications, 2009. DICTA '09. pp. 103-110 (Dec 2009)

5. Fattal, R.: Single image dehazing. In: ACM SIGGRAPH Papers. pp. 72:1-72:9. SIGGRAPH '08, ACM, New York, NY, USA (2008)

6. Fattal, R.: Dehazing using color-lines. ACM Transaction on Graphics (2014) 
7. Ferradans, S., Bertalmio, M., Provenzi, E., Caselles, V.: An analysis of visual adaptation and contrast perception for tone mapping. IEEE Transactions on Pattern Analysis and Machine Intelligence 33(10), 2002-2012 (Oct 2011)

8. Gedzelman, S.D.: Atmospheric optics in art. Appl. Opt. 30(24), 3514-3522 (Aug 1991)

9. Gibson, K., Nguyen, T.: Fast single image fog removal using the adaptive wiener filter. In: 2013 20th IEEE International Conference on Image Processing (ICIP). pp. $714-718$ (Sep 2013)

10. Hautiere, N., Tarel, J.P., Aubert, D.: Towards fog-free in-vehicle vision systems through contrast restoration. In: IEEE Conference on Computer Vision and Pattern Recognition, 2007. CVPR '07. pp. 1-8 (Jun 2007)

11. He, K., Sun, J., Tang, X.: Single image haze removal using dark channel prior. In: IEEE Conference on Computer Vision and Pattern Recognition, 2009. CVPR 2009. pp. 1956-1963 (Jun 2009)

12. He, K., Sun, J., Tang, X.: Single image haze removal using dark channel prior. IEEE Transactions on Pattern Analysis and Machine Intelligence 33(12), 2341-2353 (Dec 2011)

13. Kopf, J., Neubert, B., Chen, B., Cohen, M., Cohen-Or, D., Deussen, O., Uyttendaele, M., Lischinski, D.: Deep photo: Model-based photograph enhancement and viewing. In: ACM SIGGRAPH Asia 2008 Papers. pp. 116:1-116:10. SIGGRAPH Asia '08, ACM, New York, NY, USA (2008)

14. Koschmieder, H.: Theorie der horizontalen Sichtweite: Kontrast und Sichtweite. Keim \& Nemnich (1925)

15. Makarau, A., Richter, R., Muller, R., Reinartz, P.: Haze detection and removal in remotely sensed multispectral imagery. IEEE Transactions on Geoscience and Remote Sensing 52(9), 5895-5905 (Sep 2014)

16. McCartney, E.J.: Optics of the Atmosphere: Scattering by Molecules and Particles. Wiley (Jan 1976)

17. Middleton, W.E.K.: Vision through the atmosphere. University of Toronto Press (1952)

18. Narasimhan, S., Nayar, S.: Chromatic framework for vision in bad weather. In: IEEE Conference on Computer Vision and Pattern Recognition, 2000. Proceedings. vol. 1 , pp. 598-605 vol.1 (2000)

19. Narasimhan, S., Nayar, S.: Contrast restoration of weather degraded images. IEEE Transactions on Pattern Analysis and Machine Intelligence 25(6), 713-724 (Jun 2003)

20. Narasimhan, S.G., Nayar, S.K.: Vision and the atmosphere. International Journal of Computer Vision 48(3), 233-254 (Jul 2002)

21. Narasimhan, S.G., Nayar, S.K.: Interactive (de) weathering of an image using physical models. IEEE Workshop on Color and Photometric Methods in Computer Vision 6(6.4), 1 (2003)

22. Nayar, S., Narasimhan, S.: Vision in bad weather. In: The Proceedings of the Seventh IEEE International Conference on Computer Vision, 1999. vol. 2, pp. 820-827 vol.2 (1999)

23. Nishino, K., Kratz, L., Lombardi, S.: Bayesian defogging. Int J Comput Vis 98(3), 263-278 (Jul 2012)

24. Palma-Amestoy, R., Provenzi, E., Bertalmio, M., Caselles, V.: A perceptually inspired variational framework for color enhancement. IEEE Transactions on Pattern Analysis and Machine Intelligence 31(3), 458-474 (Mar 2009)

25. Rizzi, A., Gatta, C., Marini, D.: A new algorithm for unsupervised global and local color correction. Pattern Recogn. Lett. 24(11), 1663-1677 (Jul 2003) 
26. Sapiro, G., Caselles, V.: Histogram modification via differential equations. Journal of Differential Equations 135(2), 238-268 (Apr 1997)

27. Schaul, L., Fredembach, C., Susstrunk, S.: Color image dehazing using the nearinfrared. In: 2009 16th IEEE International Conference on Image Processing (ICIP). pp. 1629-1632 (Nov 2009)

28. Schechner, Y., Narasimhan, S., Nayar, S.: Instant dehazing of images using polarization. In: Proceedings of the 2001 IEEE Computer Society Conference on Computer Vision and Pattern Recognition, 2001. CVPR 2001. vol. 1, pp. I-325-I-332 vol.1 (2001)

29. Shiau, Y.H., Yang, H.Y., Chen, P.Y., Chuang, Y.Z.: Hardware implementation of a fast and efficient haze removal method. IEEE Transactions on Circuits and Systems for Video Technology 23(8), 1369-1374 (Aug 2013)

30. Tan, R.: Visibility in bad weather from a single image. In: IEEE Conference on Computer Vision and Pattern Recognition, 2008. CVPR 2008. pp. 1-8 (Jun 2008)

31. Tarel, J.P., Hautiere, N.: Fast visibility restoration from a single color or gray level image. In: 2009 IEEE 12th International Conference on Computer Vision. pp. 2201-2208 (Sep 2009)

32. Yoon, I., Kim, S., Kim, D., Hayes, M., Paik, J.: Adaptive defogging with color correction in the HSV color space for consumer surveillance system. IEEE Transactions on Consumer Electronics 58(1), 111-116 (Feb 2012)

33. Zamir, S., Vazquez-Corral, J., Bertalmio, M.: Gamut mapping in cinematography through perceptually-based contrast modification. IEEE Journal of Selected Topics in Signal Processing 8(3), 490-503 (Jun 2014)

34. Zhang, Y., Guindon, B.: Quantitative assessment of a haze suppression methodology for satellite imagery: effect on land cover classification performance. IEEE Transactions on Geoscience and Remote Sensing 41(5), 1082-1089 (May 2003) 